\title{
Efecto del contenido de Ni en las propiedades ópticas y eléctricas de recubrimientos ZrTiSiNiN depositados por co-sputtering
}

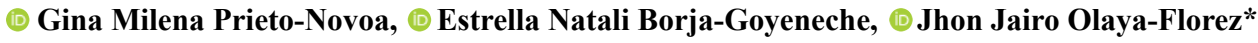 \\ Departamento de Ingeniería Mecánica y Mecatrónica, Universidad Nacional de Colombia, Sede Bogotá
}

\begin{abstract}
Resumen
Películas delgadas de ZrTiSiNiN fueron depositadas sobre sustratos de vidrio y silicio mediante co-sputtering reactivo con magnetrón usando blancos de $\mathrm{Ti}_{5} \mathrm{Si}_{2}, \mathrm{Zr}$. En esta investigación se varío el contenido de $\mathrm{Ni}$ en los recubrimientos mediante la adición de cubos de Ni ubicados sobre el blanco de Zr. La morfología superficial, la estructura cristalina y el espesor de las películas fueron evaluadas mediante microscopía electrónica de barrido (SEM), difracción de rayos X (XRD) e interferometría respectivamente. La resistividad eléctrica se midió mediante el método de cuatro puntas y sus propiedades ópticas se caracterizaron por espectroscopía ultravioleta / visible (UV/Vis). Con base en los resultados de XRD se observó que el Níquel actúa como refinador de grano al lograr disminuir el tamaño de cristalito de $27 \mathrm{~nm}$ hasta $15 \mathrm{~nm}$ cuando la concentración de Níquel aumenta de 0 at $\%$ a 6,8 at\%. Tanto la resistividad eléctrica y el "band gap" óptico de los recubrimientos aumentaron con la disminución del tamaño del cristalito como resultado del aumento de la densidad de límites de grano y del efecto de confinamiento cuántico.
\end{abstract}

Palabras clave: ZrTiSiNiN; Propiedades ópticas; Resistividad eléctrica; Sputtering.

Effect of Ni content on the optical and electrical properties of ZrTiSiNiN thin films deposited by co-sputtering

\begin{abstract}
Thin films of ZrTiSiNiN have been deposited onto a glass and silicon substrates by reactive magnetron co-sputtering of pure $\mathrm{Ti}_{5} \mathrm{Si}_{2}, \mathrm{Zr}$ alloy targets. In this investigation was located $\mathrm{Ni}$ pieces on $\mathrm{Zr}$ target in order to change the $\mathrm{Ni}$ amount in the films. The surface morphology and crystalline structure of the films were investigated by scanning electronic microscope (SEM), X-ray diffraction (XRD) and interferometry respectively. The electrical resistivity was measured by the four-point probe method and their optical properties were characterized by ultraviolet/visible (UV/ Vis) spectroscopy. XRD results showed that nickel works as a grain refiner because the crystallite size is reduced from $27 \mathrm{~nm}$ to $15 \mathrm{~nm}$ when the Ni concentration increases from 0 to $\%$ to 6.8 to $\%$. Both the electrical resistance and the optical "band gap" of the coatings increased with the decrease in crystallite size because of the increase in the density of grain boundaries and the quantum confinement effect.
\end{abstract}

Key words: ZrTiSiNiN; Optical properties; Electrical resistivity; Sputtering.

\section{Introducción}

Con el rápido desarrollo de la tecnología, la demanda de películas multifuncionales y de alta calidad se encuentra en crecimiento. Los materiales usados en sistemas microelectromecánicos (MEMS) y microoptoelectromecánicos (MOEMS) combinan un buen comportamiento mecánico junto con propiedades electrónicas y ópticas deseadas para satisfacer los requerimientos de su uso (Crone, 2008).

Los recubrimientos multicomponentes de nitruros metálicos de transición están atrayendo un interés considerable, la aleación de diferentes componentes acarrea una mejora en la funcionalidad de los recubrimientos por medio del ajuste de su microestructura (Lind, et al., 2011; Sangiovanni, 2013). En particular, con la adición de Zr a la estructura de TiN la resistencia a la corrosión y al desgaste y la dureza del recubrimiento mejoran gracias a la formación de capas estables de óxido en la superficie y a un mecanismo de fortalecimiento de solución sólida respectivamente (Lin, et al., 2010; Wang, et al., 2000). Al dopar los recubrimientos de $(\mathrm{Ti}, \mathrm{Zr}) \mathrm{N}$ con un elemento insoluble, como lo es el $\mathrm{Si}$, las propiedades como la dureza y la resistencia a la corrosión mejoran notablemente (Ebrahimi, 2012) debido a la formación de nanocompositos duros, en los cuales una fase dura amorfa de SiNx se forma en los límites de grano de (Ti,Zr)N (Saladukhin, et al., 2015a). Otra forma de obtener nanocompositos duros es mediante la adición de una fase metálica blanda al recubrimiento de nitruro (Musil, et al., 2014), fase que puede ser metálica como el Ni. Este

\footnotetext{
*Correspondencia:

Jhon Jairo Olaya-Florez; jjolaya@unal.edu.co

Recibido: 19 de febrero de 2019

Aceptado: 3 de junio de 2019

Editor: Román Castañeda
} 
elemento no interactúa químicamente con el nitrógeno y se puede ubicar en los límites del núcleo de los nitruros, actuando como un refinador de grano (Belov, et al., 2014; Zhang, et al., 2005).

Pocos son los trabajos previos relacionados con la síntesis y caracterización del recubrimiento multicomponente ZrTiSiNiN. Se conoce el trabajo donde se estudia la microestructura y resistencia a la corrosión del recubrimiento (Zr, Si, Ti)N-Ni (Borja-Goyeneche \& Olaya-Florez, 2018). Otros trabajos reportan las propiedades microestructurales y mecánicas del recubrimiento cuaternario TiZrSiN (Saladukhin, et al., 2015; Kirik, et al., 2012; Pogrebnjak, et al., 2012). En el presente trabajo se fabrican por co-sputtering reactivo recubrimientos multifásicos de $\mathrm{ZrTiSiNiN}$ variando el contenido de $\mathrm{Ni}$, con el objetivo de proporcionar información sobre el efecto del contenido de Ni sobre las propiedades eléctricas y ópticas del recubrimiento. Los resultados de esta investigación constituyen un aporte importante que puede ser usado como referencia para otros autores.

\section{Materiales y métodos}

Se fabricaron películas delgadas de ZrTiSiNiN con la técnica de co-sputtering DC-RF y depositados a temperatura ambiente sobre sustratos de vidrio y silicio (100) los cuales fueron previamente limpiados con acetona e isopropanol en ultrasonido y secados con nitrógeno. La cámara de vacío se encuentra conectada a una bomba turbo molecular y una rotativa con que se alcanza una presión base de $9,5 \times 10^{-4}$ $\mathrm{Pa}$. Se usaron blancos de $\mathrm{Zr}$ y $\mathrm{Ti}_{5} \mathrm{Si}_{2}$ con pureza del $99,95 \%$ los cuales se conectaron a una fuente DC y RF respectivamente. Sobre el área de mayor erosión del blanco de $\mathrm{Zr}$ se ubicaron cubos de $\mathrm{Ni}$ de tamaño $3 \times 3 \times 3 \mathrm{~mm}$ y pureza del $99,99 \%$, la cantidad de estos cubos se varió de 0 a 4 con el fin de aumentar el contenido de $\mathrm{Ni}$ en el recubrimiento. Se estableció un tiempo de disposición de 1 hora, tiempo necesario para obtener recubrimientos con espesores de $1 \mu \mathrm{m}$ aproximadamente. En la figura 1 se muestra un diagrama esquemático del sistema usado para la fabricación de los recubrimientos y en la tabla 1 se resumen los parámetros de fabricación empleados.

Los recubrimientos producidos se caracterizaron por medio de diferentes técnicas analíticas. Para el análisis elemental y de morfología superficial se utilizó microscopia electrónica de barrido (SEM, por sus siglas en inglés) usando un equipo TESCAN VEGA3 con un sistema de microanálisis por espectroscopía de dispersión de energía de rayos $\mathrm{X}$ (EDS, por sus siglas en inglés) con un voltaje de aceleración de $15 \mathrm{keV}$. El estudio de la microestructura se llevó a cabo por difracción de rayos X (XRD, por siglas en inglés) empleando un sistema Panalitical en modo BraggBrentano con radiación $\mathrm{Cu}-\mathrm{K} \alpha(35 \mathrm{~mA}, 40 \mathrm{kV})$ con una velocidad de escaneo de $0,02 \%$ s usando un detector tipo centelleante. Los espesores de las películas se obtuvieron mediante interferometría con el microscopio óptico 3D Bruker ContourGT-K. La rugosidad de los recubrimientos fue determinada con el perfilómetro Dektak 150 Surface Profiler. Las propiedades ópticas fueron estudiadas por espectroscopía (UV/Vis) con el espectrofotómetro Cary 5000 UV-VIS-NIR. La resistividad eléctrica de los recubrimientos se midió usando el método convencional de cuatro puntas (FPP, por sus siglas en inglés) en configuración lineal y a temperatura ambiente.

\section{Resultados y discución}

Composición química elemental. En la tabla 2 se muestra la composición química de los recubrimientos ZrTiSiNiN fabricados por co-sputtering reactivo. Se puede observar que el porcentaje de concentración atómica de $\mathrm{Ni}$ en los recubrimientos es directamente proporcional con la cantidad de cubos de Ni usados en la deposición, logrando una variación entre $0-6,8$ at $\% \mathrm{Ni}$. Con el aumento de la concentración de $\mathrm{Ni}$, las concentraciones de $\mathrm{Si}$ y $\mathrm{Ti}$ permanecen aproximadamente constantes, mientras que la concentración de $\mathrm{Zr}$ disminuye, lo que podría indicar que los átomos de Ni entran a la solución sólida de $(\mathrm{Ti}, \mathrm{Zr}, \mathrm{Si}) \mathrm{N}$ por sustitución de los átomos de $\mathrm{Zr}$ para formar la solución sólida $(\mathrm{Ti}, \mathrm{Zr}, \mathrm{Si}, \mathrm{Ni}) \mathrm{N}$.

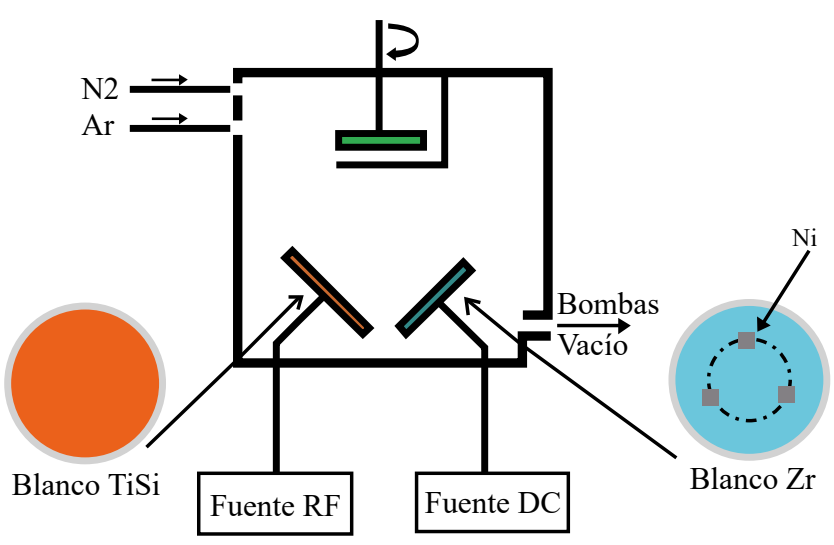

Figura 1. Diagrama esquemático sistema de deposición.

Tabla 1. Parámetros deposición películas de ZrTiSiNiN

\begin{tabular}{lc}
\hline Parámetro & Valor \\
\hline Presión base $(\mathrm{Pa})$ & $9,5 \times 10^{-4}$ \\
\hline Presión de trabajo $(\mathrm{Pa})$ & 0,4 \\
Flujo $_{2}(\mathrm{sccm})$ & 2 \\
\hline Flujo Ar $(\mathrm{sccm})$ & 14 \\
Tiempo deposición $(\mathrm{h})$ & 1 \\
\hline Potencia DC $(\mathrm{W})$ & 200 \\
Potencia $\mathrm{RF}(\mathrm{W})$ & 170 \\
Distancia sustrato-blancos $(\mathrm{m})$ & $10,2 \times 10^{-2}$ \\
Temperatura sustrato $\left({ }^{\circ} \mathrm{C}\right)$ & $28(\mathrm{ambiente})$ \\
Cantidad cubos de níquel & $0,1,2,3,4$ \\
\hline
\end{tabular}


Tabla 2. Composición elemental de las películas ZrTiSiNiN fabricadas

\begin{tabular}{cccccc}
\hline \# Cubos de Ni & Nombre muestra & Zr (at\%) & Si (at\%) & Ti (at\%) & Ni (at\%) \\
\hline 0 & ZrTiSiN-0Ni & $72,3 \pm 0,2$ & $11,8 \pm 0,4$ & $15,8 \pm 0,3$ & 0 \\
1 & ZrTiSiN-1Ni & $68,8 \pm 0,7$ & $12,7 \pm 0,5$ & $16,6 \pm 0,2$ & $2,6 \pm 0,2$ \\
2 & ZrTiSiN-2Ni & $66,2 \pm 0,5$ & $12,6 \pm 0,4$ & $16,5 \pm 0,2$ & $4,0 \pm 0,3$ \\
3 & ZrTiSiN-3Ni & $65,1 \pm 0,4$ & $12,8 \pm 0,4$ & $16,8 \pm 0,3$ & $5,4 \pm 0,1$ \\
4 & ZrTiSiN-4Ni & $62,0 \pm 0,5$ & $13,2 \pm 0,2$ & $17,6 \pm 0,2$ & $6,8 \pm 0,3$ \\
\hline
\end{tabular}

Espesor. Con el fin de obtener películas delgadas con un mismo espesor, todos los recubrimientos se fabricaron con un mismo tiempo de deposición. Para el presente trabajo se manejó un tiempo de deposición de 1 hora para obtener recubrimientos de aproximadamente $1 \mu \mathrm{m}$. El espesor medido para cada recubrimiento se puede observar en la tabla 3.

Caracterización microestructural. La figura 2 muestra los patrones XRD de las películas de ZrTiSiNiN depositadas sobre vidrio en función de la concentración de $\mathrm{Ni}$, adicionalmente también se grafican los picos de referencia para ZrN (JCPDS 78-1420) y TiN (JCPDS 87-0633). Se puede observar que los picos de los patrones DRX de los recubrimientos producidos se localizan entre los picos del $\mathrm{ZrN}$ y $\mathrm{TiN}$, con valores $2 \theta$ de $34,76^{\circ}, 40,15^{\circ}$ y $73,13^{\circ}$, correspondiendo con los planos (111), (200) y (222) de la fase $(\mathrm{Ti}, \mathrm{Zr}) \mathrm{N}$ con estructura cristalina FCC. Estos valores concuerdan con la tarjeta JCPDS 89-5214 y trabajos previos donde estudian recubrimientos de TiZrN (Kaliaraj, $\boldsymbol{e t}$ al., 2015; Chinsakolthanakorn, et al., 2012) y TiZrSiN (Uglov, et al., 2015; Saladukhin, et al., 2015b), ambos depositados por co-sputtering reactivo.

En el patrón XRD obtenido no se observan picos de $\mathrm{Si}$, Ni o de sus compuestos, indicando que éstos podrían estar en forma amorfa dentro del recubrimiento. En el caso del $\mathrm{Si}$, éste reacciona con el $\mathrm{N}$ formando una fase amorfa a-SiNx, justificando así la ausencia de este elemento en el patrón DRX. Para el caso del Ni, la ausencia de sus picos puede deberse a la formación de una fase amorfa a-SiNx(Ni) (Zhang, et al., 2005), o a la baja concentración de Ni presente en el recubrimiento ya que éste podría ser observado por DRX si supera una concentración de 39,8 at $\%$ (Wang, et al., 2013).

Los recubrimientos depositados sobre vidrio muestran una variación en la orientación preferencial en plano (111), cuya intensidad depende de la concentración de $\mathrm{Ni}$, indicando que la cristalinidad del recubrimiento depende de la concentración de $\mathrm{Ni}$ en éste. En el recubrimiento fabricado sin Ni se observa el pico del plano (111) de la fase c-(Ti,Zr)N, sin embargo al aumentar la concentración de Ni a 2,6 at\% se presenta una mejora en la cristalinidad del recubrimiento debido al aumento de las intensidades de los picos, lográndose apreciar una orientación mixta con tres picos correspondientes a los planos (111), (200) y (222) de la fase c-(Ti,Zr)N. Al seguir aumentando la concentración
Tabla 3. Espesores películas de ZrTiSiNiN

\begin{tabular}{lc}
\hline Nombre Muestra & Espesor $(\boldsymbol{\mu m})$ \\
\hline ZrTiSiN-0Ni & $1,21 \pm 0,01$ \\
\hline ZrTiSiN-1Ni & $1,19 \pm 0,01$ \\
ZrTiSiN-2Ni & $1,18 \pm 0,01$ \\
ZrTiSiN-3Ni & $1,19 \pm 0,01$ \\
ZrTiSiN-4Ni & $1,20 \pm 0,01$ \\
\hline
\end{tabular}

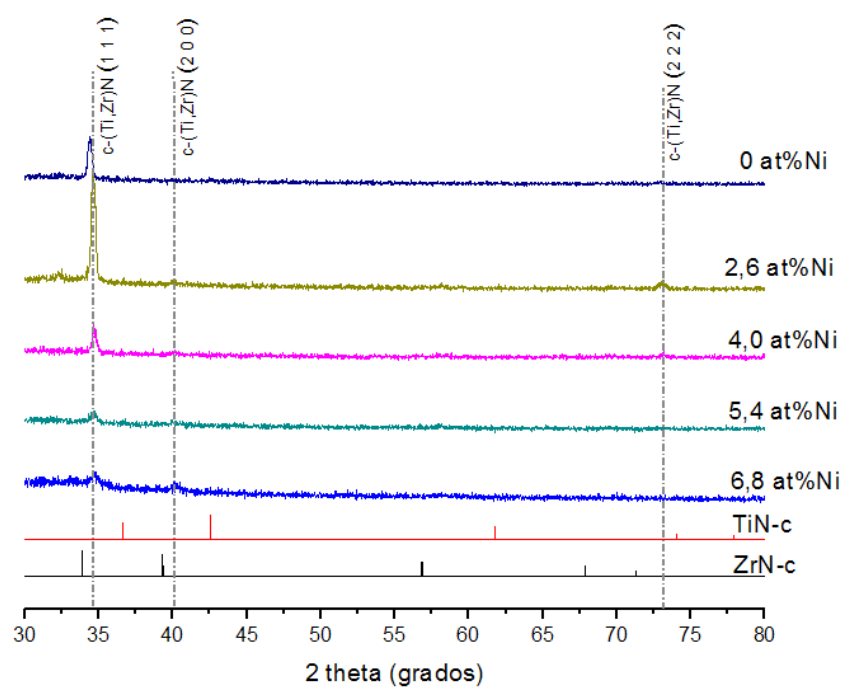

Figura 2. Patrón de difracción de rayos X de películas de ZrTiSiNiN en función de la concentración de Ni. En la parte inferior picos de referencia para ZrN-c (JCPDS 78-1420) y TiN-c (JCPDS 87-0633).

de Ni hasta un máximo de $6,8 \%$ se observa una disminución progresiva en la intensidad de los picos y un aumento su anchura, indicando una disminución de la cristalinidad de los recubrimientos.

La concentración de $\mathrm{Ni}$ en el recubrimiento tiene efecto importante sobre el tamaño de cristalito, el cual fue determinado a partir del pico (111) usando la formula Scherrer (Scherrer, 1918). En la figura 3a se grafica el tamaño de cristalito en función de la concentración de $\mathrm{Ni}$, en donde se muestra una disminución del tamaño del cristalito desde 27 $\mathrm{nm}$ hasta $15 \mathrm{~nm}$ cuando la concentración de Ni aumenta desde 0 hasta 6,8 at $\%$. Estudios previos sobre el efecto de 

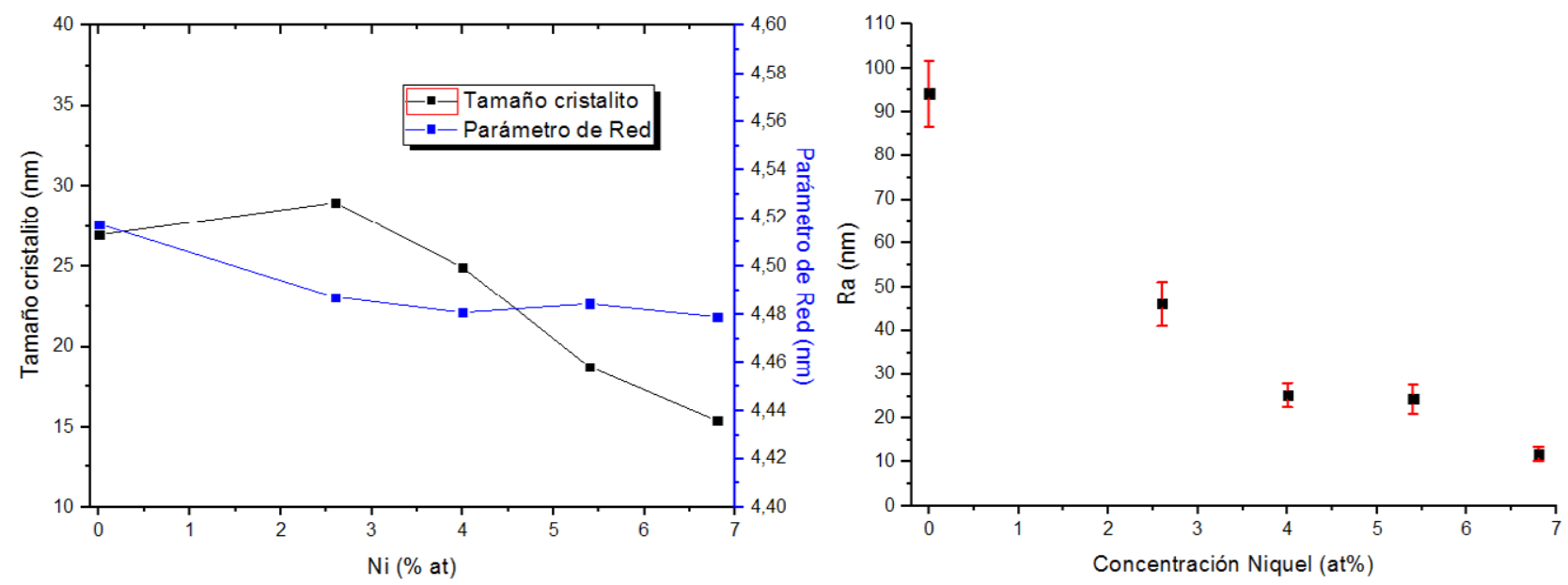

Figura 3. a) Tamaño de cristalito y parámetro de red de recubrimientos de ZrTiSiNiN en función de la concentración de Ni. b) Rugosidad promedio de recubrimientos de ZrTiSiNiN en función de la concentración de Ni.

la adición de Ni en recubrimientos de TiN (Akbari, et al., 2006), nc-TiN/a-SiNx (Zhang, et al., 2005) y nc-CrAlN/aSiNx (Wang, et al., 2013) han demostrado que el níquel funciona como un refinador de grano, en donde sus átomos forman una fase amorfa intergranular que impide el crecimiento del grano, al igual que lo hace el Si al generar la fase amorfa a-SiNx.

Además de observarse cambios en la amplitud y ancho de los picos del patrón de difracción de los recubrimientos fabricados, también se presentó un corrimiento del pico (111) hacia ángulos $2 \theta$ mayores con la adición de $\mathrm{Ni}$, en comparación con el recubrimiento sin Ni. Este corrimiento indica una disminución en el parámetro de red, tal como se puede observar en la figura3a, efecto que puede deberse por la sustitución de átomos de $\mathrm{Zr}$ por átomos de menor tamaño en este caso por átomos de Ni o Ti. Debido a que en el presente estudio la máxima concentración de Ni lograda fue de 6,8 at $\%$, no se logra observar grandes cambios en el parámetro de red en función de la concentración de Ni. Sin embargo, la dependencia del parámetro de red con la concentración de Ni se logra observar claramente en trabajos previos en los que la concentración de Ni es alta (Lindahl, et al., 2018; Zhang, et al., 2005).

Morfología superficial y rugosidad. Imágenes SEM de la morfología superficial de los recubrimientos ZrTiSiNiN con diferentes concentraciones de Níquel se representan en la figura 4. Se puede observar que el número y tamaño de los defectos superficiales en los recubrimientos disminuye con el aumento del contenido de Ni. La morfología superficial presentada por los recubrimientos fabricados coincide con la reportada en trabajos previos (Pilloud, et al., 2003), en donde recubrimientos de $\mathrm{ZrN}$ fabricados por sputtering muestran una morfología superficial con defectos tipo nodular, los cuales son propios de recubrimientos duros fabricados por sputtering (Panjan, et al., 2012) y que, principalmente, se generan en el proceso de fabricación por contaminación de partículas extrañas en la superficie del sustrato.
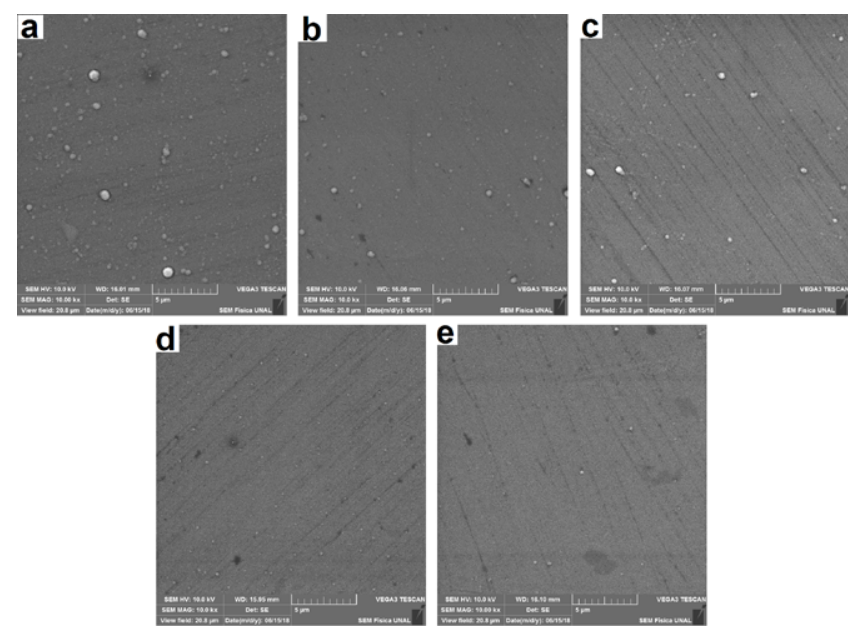

Figura 4. Imágenes SEM de la morfología superficial de los recubrimientos $\mathrm{ZrTiSiNiN}$ con diferentes concentraciones de Níquel: a) 0 at $\%$ b) 2,6 at $\%$ c) 4,0 at $\%$ d) 5,4 at $\%$ e) 6,8 at $\%$. Aumento de 10kX.

La disminución de defectos superficiales conlleva a una disminución en la rugosidad superficial de los recubrimientos, tal y como se muestra en la figura $3 \mathrm{~b}$, en donde se grafica la rugosidad promedio de los recubrimientos de ZrTiSiNiN en función de la concentración de Ni. Se observa que la rugosidad de los recubrimientos disminuye con la adición de $\mathrm{Ni}$, pasando de $94 \mathrm{~nm}$ a $12 \mathrm{~nm}$ cuando la concentración de $\mathrm{Ni}$ aumenta de 0 at $\%$ a 6,8 at $\%$. Este mismo efecto del Ni sobre la rugosidad del recubrimiento se observó en recubrimientos de $\mathrm{CrNiN}$ fabricados por sputtering (Tan, et al., 2015).

Caracterización óptica. Los espectros de reflectancia, transmitancia y absorbancia óptica de los recubrimientos de ZrTiSiNiN con diferentes concentraciones de $\mathrm{Ni}$ y depositados sobre vidrio son mostrados en la figura 5. Se puede observar que la totalidad de la radiación electromagnética 
que incide sobre los recubrimientos es absorbida, ya que la suma de la transmitancia y la reflectancia no superan el 3\%. Esta alta absorbancia puede deberse a una alta densidad en defectos intrínsecos del material (puntuales o lineales), defectos que son propios de los recubrimientos duros como los nitruros (Mayrhofer, et al., 2006) y cuya densidad aumenta con la adición de elementos aleantes y el espesor del recubrimiento. En nuestro caso, al fabricar recubrimientos multicomponentes ( 5 componentes) y con un alto espesor $(>1 \mu \mathrm{m})$, la densidad de defectos intrínsecos es alta y con ella la absorbancia del recubrimiento, ya que estos defectos actúan como centros de absorción. Es por esta razón que en la literatura se encuentran estudios relacionados con propiedades ópticas en recubrimientos con espesores que en su mayoría no superan los 300nm.

Para longitudes de ondas mayores a $1400 \mathrm{~nm}$ el aumento en la concentración de $\mathrm{Ni}$ afecta la transmitancia y absorbancia de los recubrimientos de ZrTiSiNiN, mientras que para longitudes menores no se observa un efecto claro. Para longitudes de onda mayores a $1400 \mathrm{~nm}$, la transmitancia y

absorbancia tienen un comportamiento similar al del tamaño de cristalito con la adición de $\mathrm{Ni}$, en donde al disminuir el tamaño de cristalito la transmitancia aumenta y la absorbancia disminuye. Si la longitud de onda es menor solo se observa que la transmitancia disminuye y la absorbancia aumenta cuando la concentración de $\mathrm{Ni}$ pasa de 0 at $\%$ a 2,6 at $\%$, después se mantienen aproximadamente constante independientemente del contenido de $\mathrm{Ni}$ en el recubrimiento.

Con el aumento de la concentración de $\mathrm{Ni}$ la reflectancia óptica disminuye en todo el rango de longitudes de onda estudiadas (300 nm-2500 nm). Esta disminución en la reflectancia puede deberse a la disminución de la rugosidad superficial de los recubrimientos, disminución que se logró con el aumento de la concentración de Ni. Estudios previos han demostrado que las superficies rugosas causan una mayor dispersión de la luz, lo que conduce a una mayor reflectancia (Lee, et al., 2012).

Para calcular la energía de la banda prohibida (band gap óptico) se usa la relación de Tauc (Tauc, 1974):

$$
\alpha h v=A\left(h v-E_{g a p}\right)^{n},
$$


Figura 5. Reflectancia, Transmitancia y Absorbancia en función de la longitud de onda para recubrimientos de ZrTiSiNiN con diferentes concentraciones de Ni. 
donde $\alpha$ es el coeficiente de absorción, A es una constante, $h$ es la constante de Planck, $v$ es la frecuencia del fotón, Egap es el band gap óptico y $\mathrm{n}$ es el exponente que caracteriza la transición electrónica. Para este trabajo se usó $n=1 / 2$ para analizar el caso de las transiciones directas permitidas, y se halló el coeficiente de absorción con la relación $\alpha=2.303$ $\mathrm{A} / \mathrm{t}$, siendo A la absorbancia de la película y $\mathrm{t}$ es su espesor (Mathew, et al., 2008). Así, el Egap se obtiene hallando el cruce por cero de la extrapolación lineal de la gráfica $(\alpha \mathrm{hv})^{2}$ vs hv, como se puede observar en la figura $6 \mathrm{a}$.

Los valores de energía de la banda prohibida Egap en función de la concentración de Ni son mostrados en la figura 6b. Se puede observar que el comportamiento del Egap es inversamente proporcional al presentado por el tamaño del cristalito (Figura 3a), cuando el tamaño del cristalito es mínimo el Egap es máximo, y cuando el tamaño de cristalito es máximo el Egap es mínimo. Para una concentración de 2,6 at\% Ni se observó el máximo valor para el tamaño del cristalito $(27 \mathrm{~nm})$ y el mínimo valor para el Egap $(3,7 \mathrm{eV})$, mientras que para la concentración de 6,8 at $\%$ Ni se observó el mínimo valor para el tamaño del cristalito $(15 \mathrm{~nm})$ y el máximo valor del Egap $(4,1)$.

El aumento de Egap con la disminución del tamaño del cristalito puede deberse al efecto del tamaño cuántico de las nanoestructuras del recubrimiento ZrTiSiNiN. A medida que disminuye el tamaño de los materiales a escala nanométrica se produce un confinamiento del movimiento de los electrones y huecos, conduciendo a la discretización de sus niveles de energía y un aumento en la diferencia de energía entre los estados llenos y vacíos (Singh, Goyal, \& Devlal, 2018; Smith \& Nie, 2010), es decir que el ancho de la banda prohibida aumenta al igual que lo hace su energía Egap. Trabajos previos han demostrado este mismo comportamiento que relaciona el tamaño de cristalito y el Egap en películas delgadas de SnS (Jain \& Arun, 2013), $\mathrm{TiO} 2$ (Avinash, et al., 2016) y $\mathrm{CdIn}_{2} \mathrm{Se}_{4}$ (Sudha, et al., 2016).
Caracterización eléctrica. Las curvas de voltaje en función de la corriente obtenidas a temperatura ambiente para recubrimientos de $\mathrm{ZrTiSiNiN}$ con diferentes concentraciones de Ni se muestran en la figura 7a. Se observa que todos los recubrimientos a temperatura ambiente presentan un comportamiento eléctrico lineal óhmico, cuya pendiente representa la resistencia del material.

A partir de los datos obtenidos con el método de cuatro puntas y aplicando los factores de corrección correspondientes se halla la resistividad eléctrica a temperatura ambiente de los recubrimientos ZrTiSiNiN. Las resistividades obtenidas se encuentran entre el rango de $2,88 \times 10^{4} \mu \Omega^{*} \mathrm{~cm}$ a $3,14 \times 10^{5} \mu \Omega^{*} \mathrm{~cm}$ para una variación del contenido de $\mathrm{Ni}$ entre 0 at $\%$ y 6,8 at $\%$, respectivamente.

Las resistividades obtenidas en el presente trabajo superan por cuatro órdenes de magnitud los valores de resistividades reportados en el trabajo de (Lin, et al., 2016), en donde obtuvieron recubrimientos de TiZrN con resistividad eléctrica promedio de $126 \mu \Omega * \mathrm{~cm}$ mediante sputtering DC con magnetrón desbalanceado. La alta resistividad que presentaron los recubrimientos de ZrTiSiNiN, en comparación con los de TiZrN, se debe a que tanto los átomos de Si como de Ni actúan como impurezas las cuales aumentan la dispersión de electrones conductores libres y disminuyen su camino libre medio, por lo tanto, la resistividad eléctrica del material aumenta. Trabajos previos sobre películas delgadas de $\mathrm{ZrSiN}$ (Sandu, et al., 2007; Pilloud, et al., 2006) han reportado resistividades eléctricas del mismo orden de magnitud que las halladas en el presente trabajo.

Las resistividades en función del contenido de $\mathrm{Ni}$ se muestran en la figura $7 b$, en donde se observa, como era de esperarse, el aumento de la resistividad eléctrica con el aumento de la concentración de Ni en el recubrimiento. La resistividad eléctrica en materiales nanométricos está influenciada principalmente por los límites de grano y en un menor grado por imperfecciones de la red como vacancias
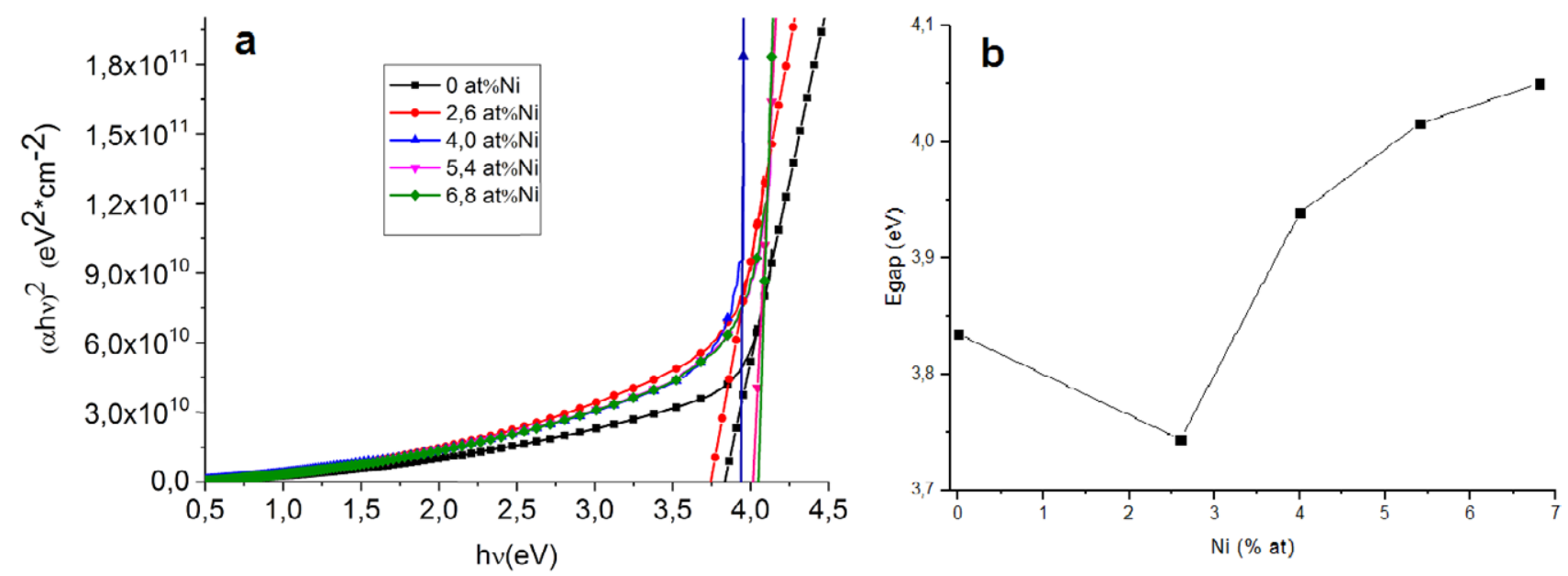

Figura 6. a) Cálculo de la banda de energía prohibida Egap para recubrimientos de ZrTiSiNiN con diferentes concentraciones de Ni. b) Energía de la banda prohibida Egap en función de la concentración de níquel de recubrimientos de ZrTiSiNiN. 

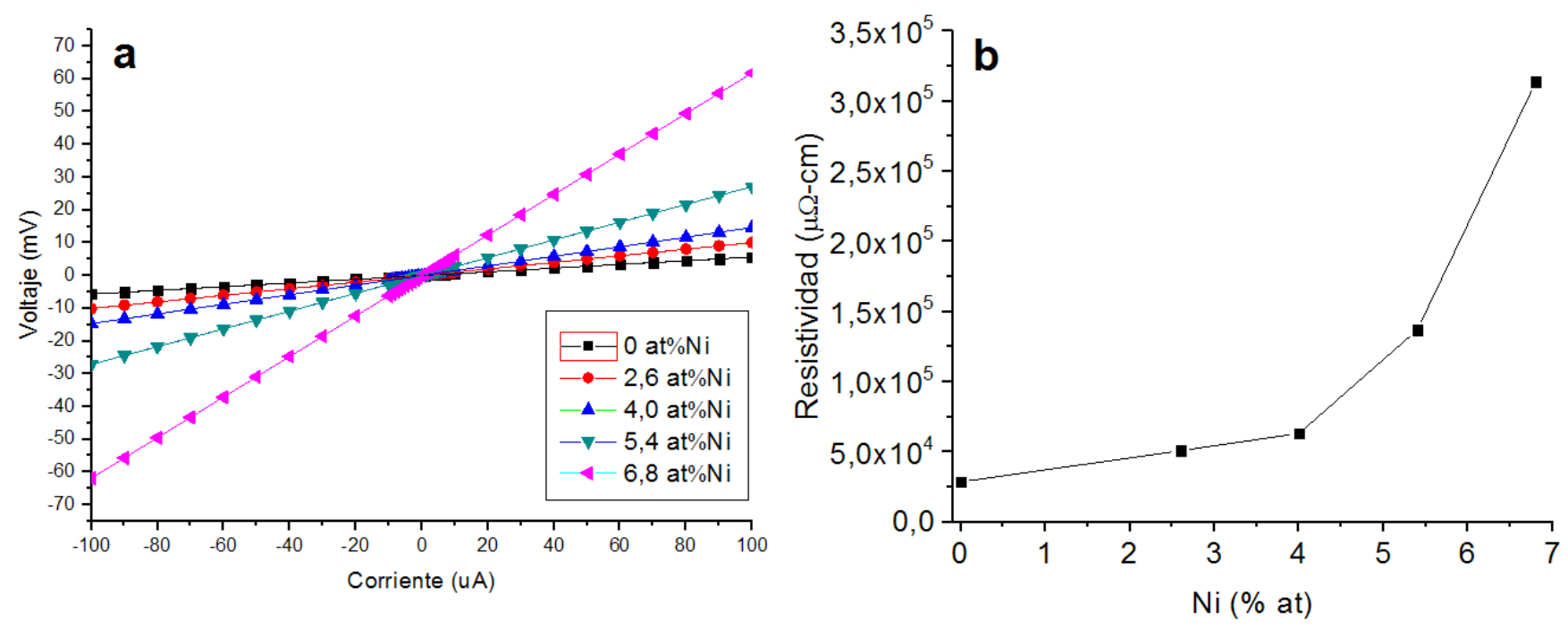

Figura 7. a) Corriente vs Voltaje para recubrimientos de ZrTiSiNiN con diferentes concentraciones de Ni. b) Resistividad eléctrica en función de la concentración de níquel de recubrimientos de ZrTiSiNiN.

y dislocaciones (Vemuri, et al., 2010). Los límites de grano actúan como centros de dispersión adicionales para los electrones de conducción (Marom, et al., 2006), por lo tanto, con la disminución del tamaño de cristalito el volumen del límite de grano aumenta, conllevando un incremento en la resistividad eléctrica.

\section{Conclusiones}

Se estudió la influencia de la adición de $\mathrm{Ni}$ en las propiedades ópticas y eléctricas de los recubrimientos de ZrTiSiNiN depositados sobre vidrio y silicio mediante la técnica de co-sputtering reactivo. La concentración de Ni se varió desde 0 at $\%$ hasta 6,8 at $\%$, rango en el que se observó que al aumentar la concentración de Ni el tamaño de cristalito disminuyo desde $27 \mathrm{~nm}$ hasta $15 \mathrm{~nm}$, corroborando el efecto del $\mathrm{Ni}$ como refinador de grano.

$\mathrm{Al}$ aumentar la concentración de $\mathrm{Ni}$ en los recubrimientos la resistividad eléctrica también aumentó desde $2,88 \times 10^{4} \mu \Omega^{*} \mathrm{~cm}$ hasta $3,14 \times 10^{5} \mu \Omega^{*} \mathrm{~cm}$, este aumento en la resistividad puede deberse a que los átomos $\mathrm{Ni}$ adicionados actúan como impurezas que disminuyen el camino libre medio de los electrones generando un aumento en la resistividad eléctrica del recubrimiento.

Se observó que la totalidad de la radiación electromagnética que incide sobre los recubrimientos de ZrTiSiNiN es absorbida. Esta alta absorción se debe a que se fabricaron recubrimientos duros multicomponente con espesores mayores a $1 \mu \mathrm{m}$, lo que conllevó una alta densidad de defectos intrínsecos, defectos que se comportan como centros de absorción.

El "band gap" óptico de los recubrimientos fabricados aumentó de 3,8 eV a 4,1 eV cuando el contenido de $\mathrm{Ni}$ incrementó desde 0 at $\%$ hasta 6,8 at $\%$. Este valor de Egap es inversamente proporcional al tamaño del cristalito del recubrimiento y es debido al confinamiento cuántico.

\section{Agradecimientos}

Se agradece el apoyo financiero recibido para llevar a cabo esta investigación por parte del Llamado Nacional para Jóvenes Investigadores e Innovadores 2016 de Colciencias. Esta investigación fue financiada por la Universidad Nacional Colombia a través del proyecto 37935.

\section{Contribución de los autores}

Gina Milena Prieto-Novoa, estudiante encargada de realizar la caracterización de los recubrimientos y la redacción del artículo; Estrella Natali Borja-Goyeneche, estudiante encargada de fabricar los recubrimientos del artículo; Jhon Jairo Olaya-Florez, docente encargado de liderar y asesorar la fabricación y caracterización de los recubrimientos, al igual que la escritura del artículo.

\section{Conflicto de intereses}

Los autores declaran no tener ningún conflicto de intereses.

\section{Referencias}

Akbari, A., Riviere, J. P., Templier, C., \& Le Bourhis, E. (2006). Structural and mechanical properties of IBAD deposited nanocomposite Ti-Ni-N coatings. Surface and Coatings Technology, 200 (22-23 SPEC. ISS.), 6298-6302. https:// doi.org/10.1016/j.surfcoat.2005.11.046

Avinash, B. S., Chaturmukha, V. S., Jayanna, H. S., Naveen, C. S., Rajeeva, M. P., Harish, B. M., ... Lamani, A. R. (2016). Effect of particle size on band gap and DC electrical conductivity of $\mathrm{TiO} 2$ nanomaterial (p. 20426). https://doi. org/10.1063/1.4946477

Belov, D. S., Blinkov, I. V., \& Volkhonskii, A. O. (2014). The effect of $\mathrm{Cu}$ and $\mathrm{Ni}$ on the nanostructure and properties of arc-PVD coatings based on titanium nitride. Surface and Coatings Technology. 260: 186-197. https://doi. org/10.1016/J.SURFCOAT.2014.09.069 
Borja-Goyeneche, E. N., \& Olaya-Florez, J. J. (2018). A microstructural and corrosion resistance study of $(\mathrm{Zr}, \mathrm{Si}, \mathrm{Ti}) \mathrm{N}-\mathrm{Ni}$ coatings produced through co-sputtering. DYNA. 85 (207): 192-197. https://doi.org/10.15446/dyna.v85n207.73304

Chinsakolthanakorn, S., Buranawong, A., Witit-Anun, N., Chaiyakun, S., \& Limsuwan, P. (2012). Characterization of nanostructured TiZrN thin films deposited by reactive DC magnetron co-sputtering. Procedia Engineering. 32: 571-576. https://doi.org/10.1016/j.proeng.2012.01.1310

Crone, W. C. (2008). A Brief Introduction to MEMS and NEMS. In Springer-Verlag (Ed.), Handbook of Experimental Solid Mechanics. Retrieved from http://citeseerx.ist.psu.edu/ viewdoc/download?doi=10.1.1.111.3275\&rep=rep1\&type=pdf

Ebrahimi, F. (2012). Nanocomposites New Trends and Developments. https://doi.org/10.5772/3389

Jain, P., \& Arun, P. (2013). Influence of grain size on the band-gap of annealed SnS thin films. Thin Solid Films. 548: 241-246. https://doi.org/10.1016/j.tsf.2013.09.089

Kaliaraj, G.S., Vishwakarma, V., Ramadoss,A., Ramachandran, D., \& Rabel, A. M. (2015). Corrosion, haemocompatibility and bacterial adhesion behavior of TiZrN-coated 316L SS for bioimplants. Bulletin of Materials Science. 38(4): 951955. https://doi.org/10.1007/s12034-015-0949-1

Kirik, G. V., Kozak, C., \& Opielak, M. (2012). Protective coatings based on Zr-Ti-Si-N their physical and mechanical properties and phase composition. Przeglad Elektrotechniczny. 88 (10 A): 319-321. Retrieved from http://www.scopus.com/inward/ record.url?eid=2-s2.0-84867220104\&partnerID=tZOtx3y1

Lee, C. H., Guo, F. G., \& Chu, C. C. (2012). The Thickness Dependent of Optical Properties, Resistance, Strain and Morphology of Mo Thin Films for The Back Contact of CIGS Solar Cells. Chinese Journal of Physics. 50 (2): 311-321.

Lin, Y.-W., Huang, J.-H., \& Yu, G.-P. (2010). Effect of nitrogen flow rate on properties of nanostructured TiZrN thin films produced by radio frequency magnetron sputtering. Thin Solid Films. 518 (FEBRUARY 2005): 7308-7311. https:// doi.org/10.1016/j.tsf.2010.04.099

Lin, Y.-W., Lu, C.-W., Yu, G.-P., \& Huang, J.-H. (2016). Structure and Properties of Nanocrystalline (TiZr) x N $1-\mathrm{x}$ Thin Films Deposited by DC Unbalanced Magnetron Sputtering. Journal of Nanomaterials. 2016: 1-12. https:// doi.org/10.1155/2016/2982184

Lind, H., Forsén, R., Alling, B., Ghafoor, N., Tasnádi, F., Johansson, M. P., ... Odén, M. (2011). Improving thermal stability of hard coating films via a concept of multi-component alloying. Applied Physics Letters. 99 (9): 91903. https://doi.org/10.1063/1.3631672

Lindahl, E., Ottosson, M., \& Carlsson, J. O. (2018). Doping of metastable $\mathrm{Cu} 3 \mathrm{~N}$ at different Ni concentrations: Growth, crystallographic sites and resistivity. Thin Solid Films. 647 (June 2017): 1-8. https://doi.org/10.1016/j.tsf.2017.12.010

Marom, H., Ritterband, M., \& Eizenberg, M. (2006). The contribution of grain boundary scattering versus surface scattering to the resistivity of thin polycrystalline films. Thin Solid Films. 510 (1-2): 62-67. https://doi.org/10.1016/j. tsf.2005.12.155

Mathew, S., Menon, C. S., \& Sudarsanakumar, C. (2008). Effect of thickness on the absorption spectra of $\mathrm{GaPcCl}, \mathrm{SnPcO}$ and $\mathrm{AlPcOH}$ thin films. Optoelectronics and Advanced Materials, Rapid Communications. 2 (6): 349-352.
Mayrhofer, P. H., Mitterer, C., Hultman, L., \& Clemens, H. (2006, November 1). Microstructural design of hard coatings. Progress in Materials Science. Pergamon. https:// doi.org/10.1016/j.pmatsci.2006.02.002

Musil, J., Zeman, P., \& Baroch, P. (2014). Hard Nanocomposite Coatings. Comprehensive Materials Processing (Vol. 4). Elsevier. https://doi.org/10.1016/B978-0-08-096532-1.00416-7

Panjan, P., Čekada, M., Panjan, M., Kek-Merl, D., Zupanič, F., Čurković, L., \& Paskvale, S. (2012). Surface density of growth defects in different PVD hard coatings prepared by sputtering. Vacuum. 86(6): 794-798. https://doi.org/10.1016/j. vacuum.2011.07.013

Pilloud, D., Dehlinger, A. S., Pierson, J. F., Roman, A., \& Pichon, L. (2003). Reactively sputtered zirconium nitride coatings: structural, mechanical, optical and electrical characteristics. Surface and Coatings Technology. 174175: $720-724$. https://doi.org/10.1016/S0257-8972

Pilloud, D., Pierson, J. F., \& Pichon, L. (2006). Influence of the silicon concentration on the optical and electrical properties of reactively sputtered $\mathrm{Zr}-\mathrm{Si}-\mathrm{N}$ nanocomposite coatings. Materials Science and Engineering B: Solid-State Materials for Advanced Technology. 131 (1-3): 36-39. https://doi.org/10.1016/j.mseb.2006.03.017

Pogrebnjak, A. D., Shpak, A. P., Beresnev, V. M., Kolesnikov, D. A., Kunitskii, Y. A., Sobol, O. V., ... Grudnitskii, V. V. (2012). Effect of Thermal Annealing in Vacuum and in Air on Nanograin Sizes in Hard and Superhard Coatings $\mathrm{Zr}-\mathrm{Ti}-\mathrm{Si}-\mathrm{N}$. Journal of Nanoscience and Nanotechnology. 12 (12): 9213-9219. https://doi.org/10.1166/jnn.2012.6777

Ramana, C. V., Smith, R. J., \& Hussain, O. M. (2003). Grain size effects on the optical characteristics of pulsed-laser deposited vanadium oxide thin films. Physica Status Solidi (A) Applied Research. 199 (1): 5-7. https://doi.org/10.1002/ pssa.200309009

Saladukhin, I. A., Abadias, G., Michel, A., Uglov, V. V., Zlotski, S. V., Dub, S. N., \& Tolmachova, G. N. (2015). Structure and hardness of quaternary TiZrSiN thin films deposited by reactive magnetron co-sputtering. Thin Solid Films. 581: 25-31. https://doi.org/10.1016/j.tsf.2014.11.020

Saladukhin, I. A., Abadias, G., Michel, A., Uglov, V. V., Zlotski, S. V., Dub, S. N., \& Tolmachova, G. N. (2015). Structure and hardness of quaternary TiZrSiN thin films deposited by reactive magnetron co-sputtering. Thin Solid Films. 581: 25-31. https://doi.org/10.1016/j.tsf.2014.11.020

Sandu, C. S., Medjani, F., \& Sanjinés, R. (2007). OPTICAL AND ELECTRICAL PROPERTIES OF SPUTTERED Zr-Si-N THIN FILMS: FROM SOLID SOLUTION TO NANOCOMPOSITE. Rev.Adv.Mater.Sci (Vol. 15). Retrieved from http://phys.mech.nw.ru/e-journals/RAMS/ no $31507 /$ sandu.pdf

Sangiovanni, D. G. (2013). Transition Metal Nitrides Alloy Design and Surface Transport Properties using Ab-initio and Classical Computational Methods. Linköping University. Retrieved from https://liu.diva-portal.org/smash/ get/diva2:617410/FULLTEXT01.pdf

Sherrer, P. (1918). Estimation of size and internal structural of colloidal particles by mean of Rontgen rays. Gottinger Nachrichten Math. Phys. 2: 98-100.

Singh, M., Goyal, M., \& Devlal, K. (2018). Size and shape effects on the band gap of semiconductor compound nanomaterials. Journal of Taibah University for Science. 12(4): 470-475. https://doi.org/10.1080/16583655.2018.1473946 
Smith, A. M., \& Nie, S. (2010). Semiconductor nanocrystals: structure, properties, and band gap engineering. Accounts of Chemical Research. 43 (2): 190-200. https://doi. org/10.1021/ar9001069

Sudha, D., Dhanapandian, S., Manoharan, C., \& Arunachalam, A. (2016). Structural, morphological and electrical properties of pulsed electrodeposited CdIn 2 Se 4 thin films. Results in Physics. 6: 599-605. https://doi.org/10.1016/j. rinp.2016.09.004

Tan, S., Zhang, X., Zhen, R., Tian, Z., \& Wang, Z. (2015). Effect of $\mathrm{Ni}$ content on $\mathrm{CrNiN}$ coatings prepared by RF magnetron sputtering. 120: $54-59$.

Tauc, J. (1974). Amorphous and Liquid Semiconductors. Springer US.

Uglov, V. V., Abadias, G., Zlotski, S. V., Saladukhin, I. A., Skuratov, V. A., Leshkevich, S. S., \& Petrovich, S. (2015). Thermal stability of nanostructured TiZrSiN thin films subjected to helium ion irradiation. Nuclear Instruments and Methods in Physics Research, Section B: Beam Interactions with Materials and Atoms. 354: 264268. https://doi.org/10.1016/j.nimb.2014.12.043
Vemuri, R. S., Bharathi, K. K., Gullapalli, S. K., \& Ramana, C. V. (2010). Effect of Structure and Size on the Electrical Properties of Nanocrystalline WO ${ }_{3}$ Films. ACS Applied Materials \& Interfaces. 2 (9): 2623-2628. https://doi. org/10.1021/am1004514

Wang, D.-Y., Chang, C.-L., Hsu, C.-H., \& Lin, H.-N. (2000). Synthesis of (Ti, Zr)N hard coatings by unbalanced magnetron sputtering. Surface and Coatings Technology. 130 (1): 64-68. https://doi.org/10.1016/S0257-8972(00)00675-7

Wang, Y. X., Zhang, S., Lee, J. W., Lew, W. S., \& Li, B. (2013). Toughening effect of Ni on nc-CrAlN/a-SiNx hard nanocomposite. Applied Surface Science. 265: 418-423. https://doi.org/10.1016/j.apsusc.2012.11.022

Zhang, S., Sun, D., Fu, Y., Pei, Y. T., \& De Hosson, J. T. M. (2005). Ni-toughened nc-TiN/a-SiNx nanocomposite thin films. Surface and Coatings Technology. 200 (5-6): 15301534. https://doi.org/10.1016/j.surfcoat.2005.08.080 